\title{
Z-Contrast Imaging of Nanostructures in the STEM
}

\author{
P. M. Voyles, ${ }^{*}$ D. A. Muller,** and J. L. Grazul**
}

*Dept. of Materials Science and Engineering, University of Wisconsin - Madison, 1509 University Ave., Madison, WI, 53706-1595; voyles@engr.wisc.edu **Bell Labs, Lucent Technologies, 700 Mountain Ave., Murray Hill, NJ 07974-0636

Nanoparticles are perhaps the simplest of nanostructures. Electron microscopy has long been used to determine the size distribution and dispersion of nanoparticles, but full use of these structures requires more detailed information, particularly about their surfaces and interfaces. Fig. 1 shows examples of two nanoparticles with detrimental surface conditions.

CdSe nanoparticles are being explored for optical applications [1], but the useful optical transitions could be quenched by an amorphous surface layer or an interface with amorphous material. Fig. 1(a) shows a CdSe nanoparticle with just such an amorphous overlayer: the crystal lattice does not extend to the far right edge of the particle. There are also interfaces with another crystalline particle (lattice fringes at top center) and with a third particle to the left which is not oriented near a zone axis.

Ferromagnetic nanoparticles may have applications in data storage or spintronics, but these applications have been hindered by the formation of non-ferromagnetic oxide surface layers. Fig 1(b) shows is a Z-contrast image of a Co nanoparticle. The bright core of the particle is magnetic $\mathrm{Co}$; the dimmer surface layer is non-magnetic $\mathrm{CoO}_{\mathrm{x}}$. This is assignment is confirmed by EELS spectrum image data. Fig 1(c) shows a spectrum from the center of the particle, showing both the $\mathrm{O}$ $\mathrm{K}$ and $\mathrm{Co}_{2,3}$ edges. Fig. 1(d) shows the ratio of the integrated intensities in the $\mathrm{O}$ and Co edges after background subtraction, which decreases toward the center of the particle. The EELS data and the ADF image do not track exactly, even after correction for sample drift. This is likely caused by beam-induced uniform oxidation of the particle. Prolonged exposure $(\sim 1 \mathrm{~min})$ to the beam eventually causes the bright central core to disappear entirely.

The Si microelectronics industry has been making nanostructures more complicated that particles for a generation, and has reached the point that even a few atoms out of place will soon have the potential to degrade the performance of a device [2]. Identifying one atom out of place is a significant challenge, but one we can meet with Z-contrast STEM. Fig 2 is a Z-contrast image of a $\mathrm{Si}$ sample doped with $\mathrm{Sb}$ during growth by low-temperature MBE. An Sb atom with $Z=51$ scatters $\sim 9$ times the high-angle intensity as a single $\mathrm{Si}$ atom with $Z=14$, so with a thin enough sample, we can see contrast from individual atoms. Fig 2 . shows the interface between the doped thin film and the undoped substrate. In the substrate, all the atomic columns are the same intensity. In the doped film, some of the atomic columns are much brighter, indicating they contain at least one $\mathrm{Sb}$ atom. Detailed analysis of the image intensity statistics indicates that the majority of atomic columns in images similar to Fig. 2 contain only one Sb atom [3]. 
Determining the full three-dimensional position of a substitutional impurity may also be possible. Fig. 3 shows that the simulated intensity of an Sb-containing column varies with the depth of the $\mathrm{Sb}$ in the column. If the sample is thin enough $(<100 \AA)$ for the $200 \mathrm{kV}$ probe used here), and the impurities are low enough density that having two or more in one column is statistically improbable, the intensity of the column is a single-valued function of the depth. If the sample is too thick, or if it
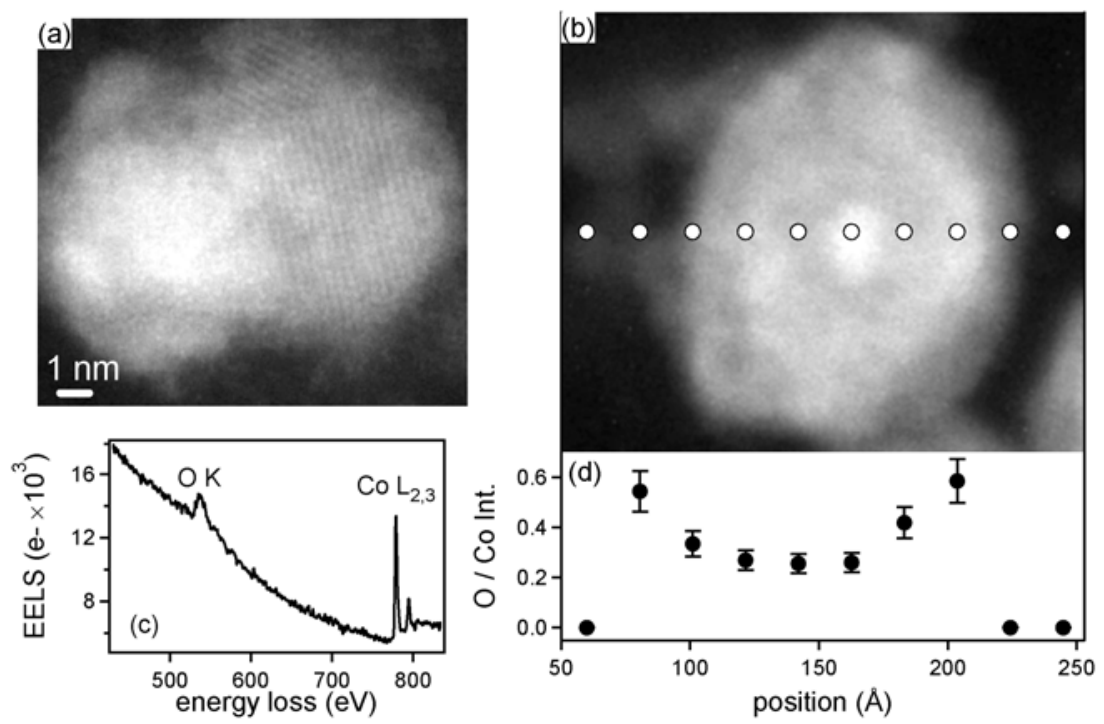

Fig. 1: (a) CdSe nanoparticle with an amorphous surface layer visible over the crystalline lattice on the left. (b) Z-contrast STEM image of a $\mathrm{CoO} / \mathrm{Co}$ nanoparticle. The Co core is brighter than the $\mathrm{CoO}_{\mathrm{x}}$ surface layer. (c) EELS data from the Co nanoparticle in (b) showing the presence of both Co and O. (d) The relative intensity of the $\mathrm{O} \mathrm{K}$ and $\mathrm{Co}_{2,3}$ edges as a function of position across the particle, extracted from a spectra taken at the positions indicated by the circles in (b). (The positions have been corrected for the measured $0.21 \AA / \mathrm{s}$ drift of the sample in the $-x$ direction.) The ratio decreases near the center of the particle, confirming that the surface is O-rich.

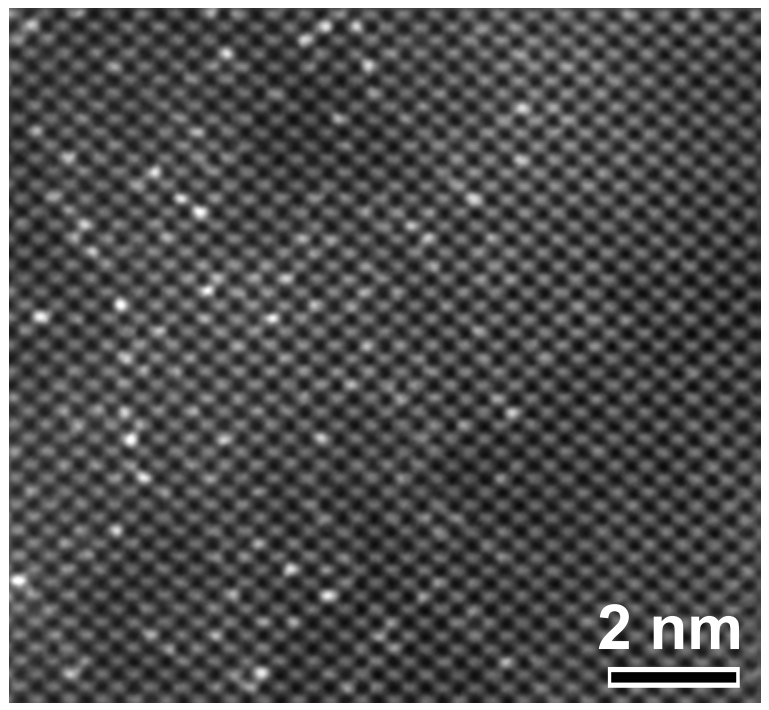
is likely that more than one impurity lies in a column, the intensity as a function of depth is no longer single-valued, and no additional information can be garnered [4].

[1] V. I. Klimov, et al., Science 290, 314 (2000).

[2] P. A. Packan, Science 285, 2079 (1999).

[3] P. M. Voyles, et al., Nature 416, 826 (2002).

[4] We thank M. Petruska, H. Kim, and V. I. Klimov for the nanoparticle samples, and $\mathrm{H}$. J. L. Gossmann for the $\mathrm{Si}$ samples.

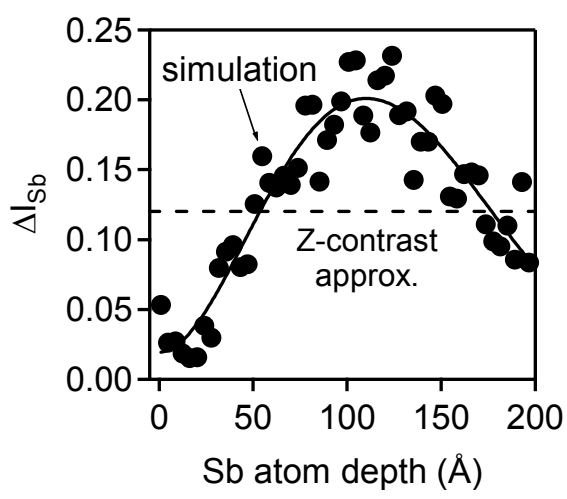

Fig. 3 Simulated excess intensity of an Sbcontaining column in $\mathrm{Si}\langle 110\rangle$, which varies with depth due to channeling. The horizontal line is the depth-independent prediction based only on atomic numbers.

Fig. 2: Z-contrast STEM image of Sb-doped Si. The right-hand side of the image is undoped substrate, in which all the atomic columns show the same intensity. The region on the right is doped. Some of the columns in this region are much brighter than the surrounding columns. Most of those columns contain just one $\mathrm{Sb}$ atom. 